\title{
El ideal de unificación en biología: el caso de la síntesis evolutiva extendida
}

\author{
The ideal of unification in biology: \\ the case of extended evolutionary synthesis
}

\begin{abstract}
Susana Gisela Lamas
Laboratorio de Investigación en Ontogenia y Adaptación (LINOA), Facultad de Ciencias Naturales y Museo. Universidad Nacional de La Plata, Argentina sglamas@yahoo.com.ar
\end{abstract}

\begin{abstract}
Resumen
En este artículo analizaré si la denominada Síntesis Evolutiva Extendida (SEE) representa una síntesis y una extensión respecto de su predecesora, la Síntesis Moderna (SM). Se argumentará que la SM propone un enfoque externalista de la evolución en tanto que la SEE considera necesario superar la dicotomía internalismo/externalismo proponiendo enfoques más integradores. Se concluirá que la SEE no puede ser considerada una extensión de la SM y que la apelación a esa extensión se relaciona con aspectos sociológicos y con el valor epistémico de la unificación teórica que siempre estuvo presente en el pensamiento evolutivo biológico.
\end{abstract}

Palabras claves: teoría evolutiva, externalismo/internalismo, perspectiva integradora, continuidad teórica, valor epistémico.

\begin{abstract}
In this article I will analyze whether the so-called Extended Evolutionary Synthesis (EES) represents a synthesis and an extension with respect to its predecessor, Modern Synthesis (MS). It will be argued that the MS proposes an externalist approach to evolution while the EES considers it necessary to overcome the internalism/externalism dichotomy by proposing more integrative approaches. It will be concluded that the EES cannot be considered an
\end{abstract}

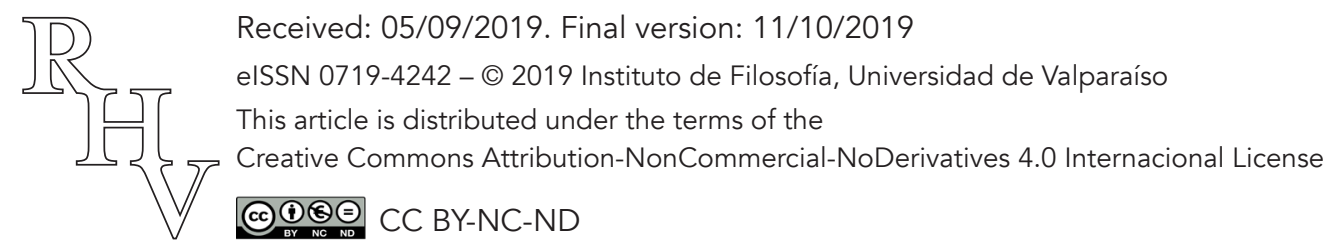


El ideal de unificación en biología: el caso de la síntesis evolutiva extendida

Susana Gisela Lamas

extension of MS and that the appeal to that extension is related to sociological aspects and the epistemic value of theoretical unification that was always present in biological evolutionary thinking.

Keywords: evolutionary theory, externalism/internalism, integrative approach, theoretical continuity, epistemic value.

\section{Introducción}

Julian Huxley en su libro La Evolución. Síntesis Moderna, describe cómo a finales del siglo XIX y principios del XX se proclamó la muerte del darwinismo a partir de la genética mendeliana y los trabajos en fisiología. A este periodo lo denomina el "eclipse" del Darwinismo ${ }^{1}$. Esta metáfora resulta interesante porque un eclipse es un ocultamiento transitorio y esto es exactamente lo que Huxley quiere conseguir con su libro, que el pensamiento de Darwin vuelva a vislumbrarse. Empezará, de este modo, un programa de investigación que conciba a la evolución a partir de los principios explicativos de la genética y la selección natural unificando sus datos y teorías; a esta unificación la denominará sintesis. En ese contexto plantea qué debería entenderse por darwinismo y lo define como una mezcla de inducción y deducción, ya que considera que fue Charles Darwin quien los aplicó por primera vez en el estudio de la evolución. Más adelante Huxley (1943) asevera que Darwin no sólo estableció el hecho de la evolución, sino que también descubrió su mecanismo sentando, así, las bases para continuar con un programa de investigación que se caracterizó por sus aspectos metodológicos y la selección natural como principio explicativo.

Cuando refiere al método de la inducción y la deducción utilizado por Darwin hace alusión a la propuesta de William Whewell en su libro History of the Inductive Sciences ${ }^{2}$. Whewell (1837) utiliza el término inducción para referirse a dos procesos distintos, el de coligación (colligation) y el de coinsilencia (consilience). Por coligación entiende el acto intelectual por el cual el entendimiento establece una conexión precisa entre los fenómenos y la caracteriza como una operación mental que permite unir muchos hechos (suprainducir) y expresarlos mediante una ley general. Mientras que a la coinsilencia la concibe como la capacidad de la hipótesis de explicar o predecir hechos diferentes a los usados cuando se la propuso. Whewell (1837) considera, no obstante, que para que exista inducción no alcanza con unificar muchos hechos, sino que debe proponerse un nuevo

\footnotetext{
${ }^{1}$ Peter Bowler (1985) retomando la noción de eclipse describe el desarrollo del pensamiento evolutivo de principios del siglo XX a cuyas teorías las caracteriza como evolucionistas y antidarwinistas.

${ }^{2}$ History of the Inductive Sciences es el primer tomo del libro Philosophy of Inductive Sciences (1840) escrito por William Whewell.
}

Revista de Humanidades de Valparaíso, 2019, No 14, 275-286

(c) $(i) \Theta(9)$ CC BY-NC-ND 
concepto que haga referencia a este principio suprainducido. Así, siguiendo la lectura que Huxley hace de Darwin, podríamos afirmar que la selección natural representaría este nuevo concepto unificador.

Sin embargo, existen otras interpretaciones acerca del método usado por Darwin en El origen, por ejemplo, Ghiselin (1969) sostiene que Darwin usó un método hipotéticodeductivo. En tanto, Mayr (2001) propone que Darwin fue pragmático y utilizó diversos métodos, en este sentido afirma:

Darwin was a pragmatist and used whatever method he thought would bring him the best results. Darwin was a very keen observer, and there is no doubt that observation was his most productive approach. However, he was also a most skillful experimenter and, particularly in his botanical researches, he conducted numerous experiments. (Mayr 2001, 498)

También existen otros enfoques donde no se enfatiza en el método utilizado sino que se considera a las redes argumentativas a partir de lo existente y lo posible y, en base a ello, se amplía el ámbito de las explicaciones legítimas racionalmente sustentables sin que deban basarse necesariamente en un método (Regner 2002).

Pero, si bien ha habido controversias en este sentido y acerca de si Darwin habría leído el libro de Whewell previamente a escribir El Origen (Ruse, 1975; Thagard, 1977), en su obra explicitó el objetivo de encontrar leyes generales que expongan los mecanismos evolutivos existentes en la naturaleza. En efecto, lo primero que se lee en El origen de las Especies es un epígrafe de Whewell acerca de la importancia de establecer leyes generales en el mundo material, y la selección natural es postulada como esa ley o mecanismo natural:

But with regard to the material world, we can at least go to far as this-we can perceive that events are brought about not by insulated interpositions of Divine power, exerted in each particular case, but by the establishment of general laws. (Whewel, citado por Darwin $1859 / 1964,2)$

No obstante, tal como afirma Smocovities (1996), para los lectores de Darwin sólo había sido establecida la facticidad de la evolución, no su mecanismo o agente causal. En este sentido la interpretación de esta autora es distinta a la proporcionada por Huxley.

Cabe señalar que Darwin, además de la selección natural, también reconoce la importancia de otros medios de modificación que los postula como leyes de la variación ${ }^{3}$, entre los que podemos enumerar a los efectos del cambio de condiciones, el uso y desuso combinados con la selección natural, la aclimatación, la variación correlativa, la

\footnotetext{
${ }^{3}$ Me refiero al capítulo quinto de On the Origin of Species.
}

Revista de Humanidades de Valparaíso, 2019, No 14, 275-286 
compensación y economía del crecimiento, etc. Esto se refleja, asimismo, en la última oración de la Introducción de El Origen, donde explicita que está convencido de que la selección natural ha sido el medio más importante, pero no el único de modificación.

\section{La Síntesis Moderna (SM)}

La lectura que Huxley (1943) hace de Darwin y que, de alguna manera, fue la heredada en los libros de textos de biología, a la que podríamos llamar la historia oficial, supone que Darwin utilizó la metodología de Whewell, llevando a cabo la unificación de datos y postulados científicos provenientes de disciplinas externas a la biología (como la geología, la geografía, etc.) y disciplinas biológicas (como la embriología y otros postulados teóricos como los del uso y desuso, variación correlativa, etc.). Julian Huxley, tal como él mismo lo explicita, se siente heredero del darwinismo y por ello, para la comprensión de la evolución, busca "armonizar" los hechos y los instrumentos de trabajo de las disciplinas tanto biológicas como no biológicas. Dentro de las primeras destaca a la genética mendeliana, la embriología, la ecología, la sistemática, la paleontología y la anatomía comparada. Entre las no biológicas reconoce la importancia del análisis matemático, la geografía y la geología. A esta nueva propuesta la denomina Síntesis Moderna y afirma que consiste, básicamente, en la unificación de los conocimientos de estos campos disciplinares a través de una mirada evolucionista en la cual la selección natural sirva como principio explicativo o mecanismo general para dar cuenta del cambio. El criterio cuantitativo de ese mecanismo lo encuentra en la genética.

Sin embargo, Mayr y Provine (1980) reconocen que las expresiones Síntesis Moderna, Teoría Neo-darwiniana y Neodarwinismo, se utilizaron para referir a diferentes modos de entender el fenómeno evolutivo ofrecidos entre 1920 y 1950 que se basaron en datos y teorías provenientes de la botánica, la sistemática, la paleontología y la genética de poblaciones. Y, tal como lo reconoce Smocovities (1996, 56-57):

The unification of biology and the emergence of evolutionary biology took place just as the centenary of the publication of Darwin's Origin was approaching. Gathering to reexamine and reassess the work of this "great man of science," evolutionary biologists and historians would begin to contribute to the burgeoning literature of Darwin studies. Rereading the present into the past, they reinvented Darwin and Darwinism as neo- Darwinism, and reinterpreted his "theory of descent with modification" as evolution by means of natural selection. Darwin was to be reconstructed once again as the "founding father" of the discipline of evolutionary biology. Yet though Darwin was to be repeatedly hailed as the Newton of biology, it was the "modern synthesis" that would function as the biological analogue of the "Newtonian synthesis" in the grand narrative of the history of science.

De esta forma, la SM queda establecida como el nuevo conjunto de teorías "herederas" de Darwin, la selección natural como su principio unificador y la genética de poblaciones 
como la disciplina que permite mostrar mediante modelos matemáticos la evolución. Asimismo, no sólo se plantea la factibilidad de la selección natural sino que queda establecida como principio mecánico causal. A partir de la SM la evolución se definirá como el cambio en la composición genética de las poblaciones, lo que conlleva a restringir los procesos evolutivos sólo a aquellos que cambian directamente las frecuencias génicas, a saber, la selección natural, la deriva, el flujo genético y la mutación. De ahí que fenómenos relacionados con las limitaciones o canalizaciones del desarrollo no se considerarán causales de procesos evolutivos debido a que no modifican directamente las frecuencias génicas.

En el proceso de desarrollo de la SM, a pesar de lo expresado por Huxley (1943), varias disciplinas fueron dejadas de lado para dar cuenta de la evolución, entre ellas la embriología que devendrá en la biología del desarrollo y la fisiología. Más adelante veremos las consecuencias que estas ausencias ocasionaron al propio darwinismo. Así, podemos advertir cómo la SM propuso un "endurecimiento" del pensamiento de Darwin, al negligir la importancia de campos disciplinares por él aceptados para entender el fenómeno evolutivo (por ejemplo, la embriología, la fisiología, etc.) y ello devino en un modo mucho más pasivo de conceptualizar a los organismos en relación al ambiente. Esta división queda claramente expresada cuando Ernest Mayr (1998) propone una diferencia entre causas próximas y remotas, afirmando que sólo estas últimas pueden dar cuenta del proceso evolutivo.

\section{El internalismo y externalismo en la biología}

Kauffman (1993), parafraseando a Monod y desde un enfoque diferente, analiza cómo el pensamiento evolutivo busca entender el orden, y asegura que en el siglo XX ha habido dos perspectivas teóricas distintas que provienen de dos tradiciones que se fundamentan en dos enfoques diferentes, el de la selección y el de la auto-organización que él pretende relacionar. En esta misma línea García Azkonobieta (2005) caracteriza así a estos enfoques:

La visión externalista intenta comprender los sistemas vivos como producto de un proceso histórico adaptativo que puede interpretarse como el cambio de las frecuencias genéticas de las poblaciones en función de las presiones selectivas externas del entorno. La visión internalista, por su lado, considera la comprensión de la organización biológica como una condición previa necesaria e ineludible para poder entender la evolución, y es más, tiende a tratar la evolución como un factor secundario, no esencial de la vida. (García Azkonobieta 2005, 9)

En este sentido, el endurecimiento de la SM antes mencionado, supuso una mayor separación entre las visiones internalista y externalista, al proponer a la selección natural como el mecanismo del cambio evolutivo considerando, por tanto, a los organismos con 
un rol mucho más pasivo que el propuesto por Darwin. Así la SM iniciada por Huxley se caracterizó por dejar de lado disciplinas como la fisiología y la embriología vinculadas con los procesos internos de los organismos, a pesar de haberse expresado en sentido contrario en su libro.

En la década de los '90 se critica severamente a la visión externalista y a los enfoques centrados en la genética. Oyama (2000a) utiliza la expresión imperialismo genético para referirse a las explicaciones biológicas evolutivas basadas en el gen y reconoce que hay otros factores que influyen en la herencia como los factores físicos, químicos, biológicos, ambientales y comportamentales que participan en el desarrollo pre y postnatal. Asevera, asimismo, que genes y entorno son parte de los sistemas de desarrollo que producen los fenotipos (Oyama 2000a). De esta manera propone entender al proceso evolutivo desde la perspectiva de los sistemas de desarrollo dentro del cual estarían incluidos los genes, es decir cambia el gen-centrismo por una perspectiva más integradora. Así Susan Oyama et al. (2001) plantean llevar a cabo explicaciones evolutivas donde se analicen los sistemas de desarrollo desde diferentes enfoques con el objetivo de proponer no una teoría entendida como modelo teórico sino un programa de investigación. En este sentido afirma:

What we have come to term developmental systems theory is not a theory in the sense of a specific model that produces predictions to be tested against rival models. Instead, it is a general theoretical perspective on development, heredity and evolution, a framework both for conducting scientific research and for understanding the broader significance of research findings. (Oyama et al. 2001, 8-9)

Es por ello que se advierte la necesidad de relacionar diferentes campos disciplinares a fin de proporcionar una red teórica o programa amplio de investigación que permita entender las múltiples causas que intervienen en los procesos de herencia y evolución donde las nociones de natura/nurtura, biología/cultura, genes/ambiente, no se entiendan de modo dicotómico (Oyama 2000b; Oyama et al. 2001). Este programa se fue extendiendo a diferentes áreas de conocimiento y, en el pensamiento evolutivo, se desarrollaron campos disciplinares novedosos como evo-devo (evolution and development), la epigenética y la construcción del nicho que plantearon una mirada integradora de los fenómenos biológicos. De esta manera, la teoría de los sistemas de desarrollo permitió una superación en los enfoques internalistas y externalistas antes mencionados proveyendo explicaciones más abarcativas de los procesos evolutivos.

\section{La Síntesis Evolutiva Extendida (SEE)}

En este contexto y reconociendo no sólo las anomalías que presentaba la SM sino el marco desde el cual esa teoría analizaba la evolución, un grupo de científicos y filósofos de la ciencia se dieron cita en el Instituto Korand Lorenz en Altenberg, Austria para realizar 
un simposio en el año 2008. Manifestaron la necesidad de proponer una nueva teoría de la evolución a la que denominaron Síntesis Evolutiva Extendida (SEE) que posee muchos aspectos en común con la Teoría de los Sistemas de Desarrollo. En la denominación de esta teoría aparecen dos términos sobre los que vale la pena detenerse síntesis y extendida. Sus autores aseveran que es una síntesis porque reconocen que han reunido datos y teorías de diversos campos disciplinares para dar cuenta del proceso evolutivo desde una perspectiva más integrativa que la SM. Y esos campos estarán representados por la epigenética, evo-devo, la plasticidad del desarrollo y la teoría de la construcción del nicho (Laland at al. 2015).

No obstante, cuando los principales representantes de la SEE argumentan acerca de por qué utilizan la noción de extensión, afirman que en la SEE mantienen los postulados de la SM aunque se enfatice en el rol que cumplen otros procesos. Así Laland et al. (2015, 1) sostienen:

The latter, which we label the 'extended evolutionary synthesis' (EES), retains the fundaments of evolutionary theory, but differs in its emphasis on the role of constructive processes in development and evolution, and reciprocal portrayals of causation. In the EES, developmental processes, operating through developmental bias, inclusive inheritance and niche construction, share responsibility for the direction and rate of evolution, the origin of character variation and organism-environment complementarity.

Por otra parte Pigliucci (2007) reconoce que sigue la propuesta de Popper al considerar que la SM es la teoría de los genes y la SEE es la teoría de las formas y que se necesita de ambas para entender los procesos evolutivos, y de allí la necesidad de unificarlas. El problema de este argumento radica en que asimila la genética a la genética de poblaciones y esa identificación, en la actualidad, no es correcta; puesto que, la información proveniente de la genética ha influido en el pensamiento evolutivo más allá de la genética de poblaciones por ejemplo con Evo-devo o el Proyecto Genoma. Además la SM se basa en ciertos prerrequisitos, como la gradualidad, el adaptacionismo, la selección natural y el gencentrismo. Todos ellos contrarios a los prerrequisitos de la SEE (Müller 2017).

Si la SEE no posee un enfoque externalista sino que intenta superar estos enfoques dicotómicos y no es una teoría basada en la genética sino en una perspectiva más integradora para explicar la evolución, cabría preguntarnos por qué se la denomina extendida. ¿Qué se extiende? Para responder a estas preguntas primero analizaremos algunas de las afirmaciones de Laland et al. $(2015,3)$ :

We conclude that the EES is not just an extension of the MS but a distinctively different framework for understanding evolution, which, alongside more traditional perspectives, can be put to service constructively within the field.

Revista de Humanidades de Valparaíso, 2019, No 14, 275-286 
Parecería ser contradictorio denominar a una teoría SEE y afirmar, por otro lado, que no es una extensión. ¿Por qué llamarla así, entonces? Considero que hay dos razones diferentes, una de orden sociológica y otra epistémica. Analizaré cada una por separado.

Llamaré razones sociológicas a aquellas relacionadas con los aspectos vinculados con la comunidad científica. En la comunidad científica hay diferentes criterios de credibilidad y de autoridad que se traducen no sólo en qué opiniones recibirán más crédito y gozarán de una mayor aceptación; sino también en los criterios editoriales para evaluar los trabajos en revistas científicas especializadas y en los grupos y líneas de pesquisa que recibirán los susidios de investigación. En el caso del pensamiento evolutivo los científicos formados en la SM siguen manteniendo gran parte de esa autoridad y, en mi opinión, esa es una de las razones por las que los proponentes de la SEE argumentan a partir de la continuidad y no de la ruptura conceptual. Este fenómeno ya ha sucedido otras veces con la SM, un ejemplo es el análisis de Gould (2004) acerca del endurecimiento que tuvo la SM respecto al adaptacionismo:

The community of evolutionary biologists is sufficiently small, and sufficiently stratified - a few lead and many follow, as in most human activities - that we need not necessarily invoke some deep and general scientific or societal trend to explain a change in opinion by a substantial community of evolutionists in different nations. A reassessment by a few key people, bound in close contact and mutual influence, might trigger a general response. The three leading exponents of hardening in America-Dobzhansky, Simpson, and Mayrworked together as colleagues in a "New York Mafia" centered at Columbia University and the American Museum of Natural History. (Gould 2004, 543)

Por otro lado, ya ha sido ampliamente discutido en la bibliografía filosófica el rol de la comunidad y los obstáculos para aceptar los cambios científicos. Y, en cierto sentido, aceptar la selección natural como mecanismo evolutivo por parte de los defensores de la SM y de la SEE da una idea de continuidad entre las teorías que permite interpretar de un modo acumulativo sus cambios conceptuales. Esto, de algún modo, está relacionado con el segundo factor antes mencionado, el epistemológico.

A lo largo de este artículo se expuso cómo en las diferentes teorías darwinistas se argumenta a favor de la nueva propuesta teórica apelando a la unificación. En el pensamiento evolutivo el ideal de unificación estuvo presente desde Darwin, proponiendo que cada teoría comprende a las demás y agrega nuevos postulados.

No obstante, lo que ha sucedido en diversas ocasiones es que se mantiene el término pero se modifica su significado, por lo cual la nueva teoría no refiere a lo mismo que la anterior. Pero, al utilizar los mismos términos y enseñar en los libros de textos este enfoque unificador, las rupturas se desvanecen. Esto puede verse, por ejemplo, con la noción de selección natural como la supervivencia del más apto en Darwin y como reproducción 
diferencial en la SM. Se habla de dos procesos diferentes, relacionados con distintos niveles de organización y con principios explicativos distintos. Pero parecería haber una continuidad entre uno y otro.

Lo mismo sucede con las denominadas Teorías Neo-Lamarckianas cuando aluden a caracteres adquiridos. Para Lamarck (1809) estos caracteres están estrechamente unidos al uso y desuso y son fenotípicos. En la actualidad no se apela al uso y desuso y uno de los indicadores de herencia epigenética son los grupos metilos que no representan un carácter fenotípico. Es decir, la utilización de un mismo término no es condición suficiente para afirmar que exista continuidad entre dos propuestas teóricas.

De algún modo considero que algo similar está sucediendo en la discusión entre la SM y la SEE, sus supuestos son diferentes. No obstante, los proponentes de la SEE señalan que hay procesos evolutivos que potencialmente pueden poseer la misma importancia que la selección natural. Considero que esta apelación constante a la selección natural es lo que permite interpretar a la SEE como una extensión de la SM; porque habría principios o supuestos explicativos que se mantienen. Y esa, en mi opinión, es la razón epistémica para entenderla en términos de unificación y acumulación cognitiva por autores que defienden a una u otra teoría.

Pigliucci y Müller (2010) no estarían de acuerdo con esta última interpretación. Ellos proponen un modelo que grafican en forma de elipses para concebir el cambio conceptual evolutivo que lo representan como una continua expansión de la teoría evolutiva en un diagrama con elipses inclusivas que comienza con tres principios de Darwin: Variación, herencia y selección natural. Continúa con los postulados de la SM: mutación genética, herencia mendeliana, genética de poblaciones, contingencia, especiación y tendencias. Y, finaliza con los de la SEE: evo-devo, plasticidad y acomodación, construcción del nicho, herencia epigenética, teoría del replicador, evolvabilidad, selección a multinivel y evolución genómica.

Pero algunas de estas disciplinas parten de supuestos distintos y, en algunos casos, contradictorios. Es importante tomar esto en consideración porque no se trata sólo de incluir a disciplinas o prácticas diversas sino que también es necesario reflexionar acerca de sus enunciados teóricos. Por ejemplo, si analizamos la genética de poblaciones en relación con la selección multinivel, la primera afirma el cambio en las frecuencias génicas (reproducción diferencial) como un indicador de la selección natural. Pero esto no sería aceptable para la selección a multinivel. Luego, entender al cambio acumulando disciplinas podría llevarnos a la aceptación de principios explicativos inconsistentes entre sí. 


\section{Conclusiones}

La SM propone a la selección natural como la principal fuerza evolutiva con un enfoque gen centrista y una estrecha vinculación con el adaptacionismo. Este último, a su vez, supone un enfoque gradual del cambio, reduccionista y externalista, en el cual el organismo es pasivo frente a los cambios ambientales.

La SEE, por otro lado, intenta vincular los enfoques internalista y externalista superando su modo dicotómico de comprender los procesos evolutivos. De esta manera, propone analizar los cambios a partir de una interrelación organismo-ambiente donde el ambiente modifica al organismo pero también el organismo modifica al ambiente. Uno de los pilares fundamentales de los procesos evolutivos es la herencia porque permite entender el cambio inter-generacional, proporcionando una perspectiva histórica. En este sentido, la SEE acepta cuatro sistemas de herencias que están vinculados a los campos disciplinares que desarrollaron esta teoría: el genético, el epigenético, el ambiental y el comportamental. Claramente los tres últimos no son aceptados por la SM pero ¿qué podemos decir respecto al primero? Los aspectos genéticos en la SEE fueron desarrollados fundamentalmente por los científicos pertenecientes a Evo-devo quienes llegaron a conclusiones bastante diferentes a las obtenidas por la SM. Esta última reconoce la necesidad de que exista variabilidad genética para explicar los cambios evolutivos. Evodevo, en cambio, considera que hay conservación de genes y que los mismos genes regulatorios son compartidos por animales con diferente plan corporal (ej: insectos y vertebrados). Y, además, que esos genes tienen roles conservados en el desarrollo.

Es por todo lo anteriormente dicho que considero que la SEE es una síntesis pero no es una extensión de la SM. Y, quizás, cuando las relaciones de poder sean modificadas en la comunidad científica, se apelará a los principios explicativos de la SEE - ya sean los actuales o algunos otros que se propongan en un futuro- sin necesidad de apelar a la selección natural como fuerza evolutiva.

\section{Agradecimientos}

Agradezco al Dr. Vicente Dressino por sus comentarios y ayuda. Esta investigación es llevada a cabo con el subsidio del Proyecto de Incentivos 11/N907 de la Universidad Nacional de La Plata.

\section{Referencias bibliográficas}

Bowler, P. (1985). El eclipse del darwinismo: teorías evolucionistas antidarwinistas en las décadas en torno a 1900. Barcelona: Ed. Labor.

Darwin, C. (1859/1964). On the Origin of Species. A Facsimile of the First Edition. Cambridge: Harvard University Press.

Revista de Humanidades de Valparaiso, 2019, No 14, 275-286

(c) (1) @) 9 CC BY-NC-ND 
El ideal de unificación en biología: el caso de la síntesis evolutiva extendida

Susana Gisela Lamas

García Azkonobieta, T. (2005). Evolución, desarrollo y (auto)organización. Un estudio sobre los principios filosóficos de la evo-devo. Tesis doctoral. Donostia-San Sebastián: Universidad del País Vasco.

Ghiselin, M. T. (1969). The Triumph of the Darwinian Method. Berkeley: University of California Press.

Gould, S. J. (2004). The Structure of Evolutionary Theory. Massachusetts y Londres: The Belknap Press of Harvard University Press Cambridge.

Huxley, J. (1943). The Modern Synthesis. London: George Allen \& Unwin L.T.D.

Kauffman, S. (1993). The origins of order: self-organization and selection in evolution. Oxford: Oxford University Press.

Laland, K. N., Uller, T., Feldman, M. W., Sterelny, K., Müller, G. B., Moczek, A., Jablonka, E., Odling-Smee, J. (2015). The extended evolutionary synthesis: its structure, assumptions and predictions. Proc. R. Soc. B., 282(1813). doi: http://doi.org/10.1098/rspb.2015.1019

Lamarck, J. B. (1809). Philosophie zoologique. Paris: Dentu.

Mayr, E. (2001). The philosophical foundations of darwinism. Proceedings of the American Philosophical Society, 145(4): 488-495.

Mayr, E., Provine W.B (1980). The Evolutionary Synthesis: Perspectives on the Unification of Biology. Cambridge: Cambridge University Press.

Mayr, E. (1998). This Is Biology: The Science of the Living World. Cambridge, MA: Harvard University Press.

Müller, G.B. (2017). Why an extended evolutionary synthesis is necessary? Interface Focus 7: 20170015. doi: http://dx.doi.org/10.1098/rsfs.2017.001

Oyama, S. (2000a). Evolution's Eye: A Systems View of the Biology-Culture Divide. Durham, NC: Duke University Press.

Oyama, S. (2000b). The Ontogeny of Information: Developmental Systems and Evolution. Durham, NC: Duke University Press.

Oyama, S., Grifiths, P. E., Gray, R. D. (2001). Cycles of Contingency. Developmental Systems and Evolution. Massachusetts: The MIT Press.

Pigliucci, M. (2007). Do We Need an Extended Evolutionary Synthesis? Evolution, 61(12): 27432749. doi: https://doi.org/10.1111/j.1558-5646.2007.00246.x

Pigliucci, M., Müller, G. B. (2010). Evolution: The Extended Synthesis. Massachusetts: The MIT Press.

Regner, A. C. (2002). O «jogo do atual e do possível» e as manhas da razão: un estudo de caso. En F. Tula Molina y P. Lorenzano (eds.), Filosofía e historia de la ciencia en el Cono Sur. Quilmes. Argentina: Editorial UNQ.

Revista de Humanidades de Valparaíso, 2019, No 14, 275-286

(c) (1) $(9)$ CC BY-NC-ND 
Ruse, M. (1975). Darwin's debt to philosophy: An examination of the influence of the philosophical ideas of John. W. Herschel and William Whewell on the development of Charles Darwin's theory of Evolution. Stud. Hist. Phil. Sci., 6(2): 159-181. doi: https://doi.org/10.1016/00393681(75)90019-9

Smocovities, V. B. (1996). Unifying Biology. The Evolutionary Synthesis and Evolutionary Biology. Princeton, New Jersey: Princeton University Press.

Thagard, P. R. (1977). Discussion Darwin and Whewell. Stud. Hist. Phil. Sci., 8(4): 353-356. doi: https://doi.org/10.1016/0039-3681(77)90026-7

Whewel, W. (1837). History of the Inductive Sciences. From the Earliest to the Present Times. London: J. W. Parker.

Whewel, W. (1840). Philosophy of the Inductive Sciences. Founded Upon Their History. London: J. W. Parker. 